\title{
SOME DETERMINANTS OF MORPHOLOGY AND RELEASE RATE FROM POLY(L)LACTIC ACID MICROSPHERES
}

\author{
A. Kishida' ${ }^{1}$, J.B. Dressman ${ }^{2}$, S. Yoshioka ${ }^{1, *}$, Y. Aso ${ }^{1}$ and Y. Takeda' \\ 'Division of Drugs, National Institute of Hygienic Sciences, Tokyo 158 (Japan) \\ ${ }^{2}$ The University of Michigan, Ann Arbor MI 48104 (U.S.A.)
}

(Received July 18, 1989; accepted in revised form January 19, 1990)

Keywords: microspheres; poly(L)lactic acid; sudan III; etoposide; evaporation rate drug loading; drug lipophilicity

Morphology and release rate from poly(L)lactic acid microspheres were studied as a function of drug loading, drug lipophilicity and solvent evaporation rate. Sudan III was used to exemplify lipophilic compounds, while etoposide served as a relatively polar drug. Drug loading was found to be an important determinant of morphology, with increased formation of distorted microspheres at higher loadings. In the case of the polar etoposide, this translated into a greater release rate. For Sudan III, however, negligible release occurred despite morphological changes, suggesting that the drug lipophilicity is also important as a determinant of release rate. When gelatin was used as the emulsifying agent, the percentage of distorted microspheres and the release rate were highly dependent on the rate of solvent evaporation. When smooth microspheres were produced with polyvinyl alcohol as the emulsifying agent, drying rate was found to be of less importance.

\section{INTRODUCTION}

One approach to achieving prolonged duration of drug therapy is to incorporate the drug into polymer microspheres for subcutaneous or intramuscular injection. Many patents and research articles describing the manufacture and release characteristics of this type of dosage form have been published. It has been reported that drug release characteristics from this type of dosage form depend largely on the preparation method [1]. Many factors, such as the nature of polymer, drug, solvent and, if used, emulsifier, determine drug release characteristics $[2,3]$. When we try to prepare microspheres with desired release rates for a given

*To whom correspondence should be addressed. drug, we usually face the challenge of selecting an appropriate combination of these factors. It is therefore important to clarify the effect of these interrelated factors in designing controlled release microspheres. In this investigation we focus on the importance of drug lipophilicity, drug loading and solvent evaporation rate in determining the morphology and release rates from poly $(\mathrm{L})$ lactic acid microspheres.

Poly (L) lactic acid was chosen because, as a biodegradable polymer, it is especially attractive for use in injectable formulations. The (L) form, rather than the racemic mixture, was chosen to avoid the handling problems and fast decomposition rate of the latter. Additionally, we chose to study not-too-low molecular weight material with relatively small degradability, so that we could focus on factors affecting release 
by mechanisms other than polymer decomposition. Sudan III was chosen to represent highly lipophilic materials, while etoposide provided an example of a relatively polar drug.

\section{MATERIALS AND METHODS}

\section{Materials}

Poly(L)lactic acid [P(L)LA], molecular weight 10,000 dalton, was synthesized from $L-$ lactic acid (C.C.A., The Netherlands) by the ring opening polymerization method [4]. For purification, the polymer obtained was dissolved in methylene chloride and precipitated by adding 10 times the volume of methanol. The polydispersity index, $M_{\mathrm{w}} / M_{\mathrm{n}}$, was calculated to be 2.4 from gel permeation chromatography. Etoposide was obtained from Nippon Kayaku (Japan), while Sudan III was purchased from Nakarai (Japan). Methylene chloride was of reagent grade from Wako Pure Chemical Industries Ltd. (Japan).

\section{Methods}

\section{Manufacture of microspheres}

Drug and polymer were first dissolved in methylene chloride. This solution was then added to an aqueous medium stirred at room temperature. The rate of stirring (300-500 rpm) and the volumes of methylene chloride and aqueous medium $(10-20 \mathrm{ml}$ and $150-250 \mathrm{ml}$, respectively) were adjusted to suit the batch size (amount of $P(L) L A$ ), which ranged from 0.5 to 2.2 g. Polyvinyl alcohol (PVA; $2 \%$ ) or gelatin (1\%) was used as the emulsifying agent, with 3 $M$ saline added in the case of etoposide batches to prevent leaching into the aqueous medium. The methylene chloride was then removed by stirring for (a) $16 \mathrm{~h}$ at atmospheric pressure, (b) $3 \mathrm{~h}$ under low vacuum (ca. $20 \mathrm{mmHg}$ ) or (c) $1 \mathrm{~h}$ under high vacuum ( $<5 \mathrm{mmHg}$ ), with (b) as the standard condition. When evaporation was complete, the microspheres were washed with cold water and sieved into seven size fractions: $<44 \mu \mathrm{m}, 44-88,88-105,105-125$, $125-149,149-177$ and $>177 \mu \mathrm{m}$, rinsed again and freeze-dried.

\section{Characterization}

Yield, particle size distribution, and percentage of drug incorporation as a function of particle size fraction were determined for each batch. Particle size distribution was determined by measuring the weight of each fraction of sieved microspheres. The 44-88 $\mu \mathrm{m}$ particle size fraction was further characterized by scanning electron microscopy (SEM) and determination of the release rate into aqueous media. For selected batches, the surface area per gram $\left(\mathrm{m}^{2} / \mathrm{g}\right)$ of the $44-88 \mu \mathrm{m}$ particle size fraction was also measured.

\section{Scanning electron microscopy}

Samples were prepared by sputter coating with $\mathrm{Au} / \mathrm{Pd}$ in an Eiko (Japan) IB-3 ion coater at $40 \mathrm{~mA}$ for ca. $2 \mathrm{~min}$, at a pressure $<0.2$ Torr. Micrographs were obtained using a Hitachi (Japan) S-450 SEM at an intensity of $15 \mathrm{kV}$ under various magnifications.

\section{Surface area}

Surface area was determined by the nitrogen adsorption/desorption method, using helium as the carrier gas, in a Micromeritics Flowsorb 2300 (Micromeritics, U.S.A.). These determinations were made in triplicate.

\section{Assay}

The drug content of microsphere was assayed by dissolving a known mass of sample in methylene chloride and comparing the absorbance at $508 \mathrm{~nm}$ (Sudan III) or $292 \mathrm{~nm}$ (etoposide) with that of a standard solution. $P(L)$ LA did not interfere with either assay.

\section{Release rate}

Release rate into water (etoposide) or Tween $801 \%$ aqueous solution (Sudan III) was determined at $37^{\circ} \mathrm{C}$ in a constant temperature water 
bath. Samples were removed at appropriate times up to $25 \mathrm{~h}$ (etoposide) or one week (Sudan III), with volume replacement of the removed sample. Assay was at $283 \mathrm{~nm}$ for etoposide and $508 \mathrm{~nm}$ for Sudan III. HPLC assay of the sample solution according to the previously reported method [5] indicated that no degradation of etoposide occurred under these conditions. All release rates were determined in duplicate or triplicate.

\section{RESULTS AND DISCUSSION}

Table 1 summarizes the manufacturing conditions, yields, batch assay results and porosities for the 11 sets of batches of P(L)LA microspheres. Figure 1 illustrates the particle size distributions and Fig. 2 shows the release rates for the batches containing etoposide. None of the Sudan III batches released any drug within the one week test period. Only when the $\mathrm{pH}$ of the medium was changed to ca. 12 with $\mathrm{NaOH}$ to accelerate decomposition of the polymer was release of Sudan III observed. Figures 3 and 4 consist of representative SEM photographs of various etoposide and Sudan III batches.

\section{Drug loading}

\section{Etoposide}

Morphology and release rate from etoposide microspheres were studied at $1 \%, 10 \%$ and $20 \%$ loadings, with solvent evaporation under low vacuum conditions. A $1 \%$ loading of etoposide produced microspheres free from surface imperfections (Fig. 3A). These microspheres did not release any drug within the $5 \mathrm{~h}$ test period

TABLE 1

Characteristics of Sudan III and etoposide-containing poly (L) lactic acid microspheres

\begin{tabular}{|c|c|c|c|c|c|c|c|}
\hline Drug & $\begin{array}{l}\text { Loading } \\
\text { (\%) }\end{array}$ & $\begin{array}{l}\text { Solvent } \\
\text { evaporation }\end{array}$ & Emulsifier & $\begin{array}{l}\text { Yield } \\
(\%)\end{array}$ & $\begin{array}{l}\text { Drug recovery } \\
(\%)^{\circ} \\
(44-88 \mu \mathrm{m})\end{array}$ & $\begin{array}{l}\text { Surface area } \\
\left(\mathrm{m}^{2} / \mathrm{g}\right)\end{array}$ & $\begin{array}{l}\text { Distorted } \\
\text { particles } \\
\text { (SEM) }\end{array}$ \\
\hline \multirow[t]{5}{*}{ Etoposide } & 1 & low vac. & $\begin{array}{l}\text { gelatin } 1 \% \\
3 \mathrm{M} \mathrm{NaCl}\end{array}$ & 80.6 & 78 & 0.263 & - \\
\hline & 10 & atmos. press. & $\begin{array}{l}\text { gelatin } 1 \% \\
3 \mathrm{M} \mathrm{NaCl}\end{array}$ & 85.9 & 87 & 1.73 & ++ \\
\hline & 10 & low vac. & $\begin{array}{l}\text { gelatin 1\% } \\
3 \mathrm{M} \mathrm{NaCl}\end{array}$ & 81.8 & 85 & 0.317 & + \\
\hline & 10 & high vac. & $\begin{array}{l}\text { gelatin } 1 \% \\
3 \mathrm{M} \mathrm{NaCl}\end{array}$ & 86.3 & 85 & 0.357 & + \\
\hline & 20 & low vac. & $\begin{array}{l}\text { gelatin } 1 \% \\
3 \mathrm{M} \mathrm{NaCl}\end{array}$ & 87.0 & 100 & 1.26 & ++ \\
\hline \multirow[t]{6}{*}{ Sudan III } & 1 & atmos. press. & PVA $2 \%$ & 81.1 & 95.4 & N.D. & - \\
\hline & 1 & low vac. & PVA2\% & 81.8 & 94.3 & N.D. & - \\
\hline & 1 & high vac. & PVA2\% & 82.2 & 88.9 & N.D. & - \\
\hline & 1 & low vac. & $\begin{array}{l}\text { gelatin } 1 \% \\
3 \mathrm{M} \mathrm{NaCl}\end{array}$ & 80.5 & 92.4 & 0.318 & - \\
\hline & 1 & atmos. press. & $\begin{array}{l}\text { gelatin } 1 \% \\
3 \mathrm{M} \mathrm{NaCl}\end{array}$ & 79.6 & N.D. & 2.47 & + \\
\hline & 10 & low vac. & $\begin{array}{l}\text { gelatin 1\% } \\
3 \mathrm{M} \mathrm{NaCl}\end{array}$ & 82.4 & 91.3 & N.D. & + \\
\hline
\end{tabular}

${ }^{a} D_{\text {rug }}$ A4-88 $\mu \mathrm{m}_{\mathrm{m}} /$ Drug $_{\text {theor }} \times 100$.

N.D.: not determined. 

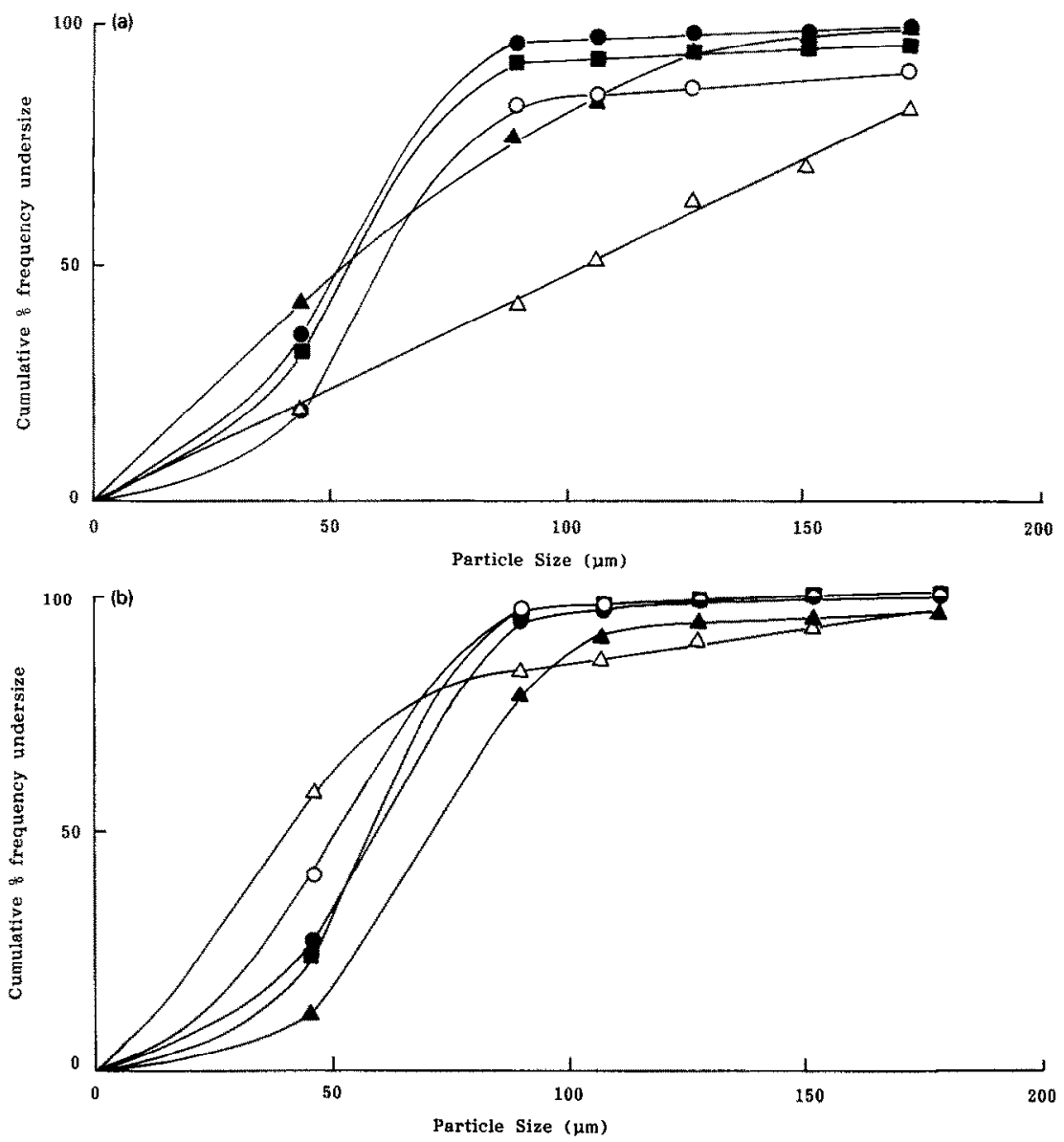

Fig. 1. Cumulative particle size distributions for P(L)LA microspheres containing etoposide (a) and Sudan III (b). (a):

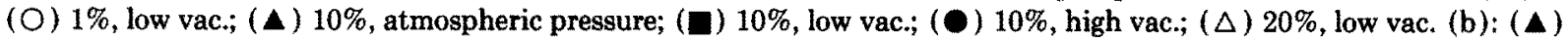
$1 \%$, PVA $2 \%$, atmospheric pressure; $(\boldsymbol{G}) 1 \%$, PVA $2 \%$, low vac.; $(O) 1 \%$, PVA $2 \%$, high vac.; $(O) 1 \%$, gelatin $1 \%$, low vac.; $(\Delta) 10 \%$, gelatin $1 \%$, low vac.

(Fig. 2). As the drug loading increased, the percentage of distorted microspheres also increased, with the $20 \%$ batch consisting almost entirely of distorted microspheres (Fig. 3d). The release rate also increased with drug loading, with a small amount of release at $10 \%$ loading and $60 \%$ release within $5 \mathrm{~h}$ at $20 \%$ loading (Fig. 2). Yields and percentages of theoretical concentration of drug in the microspheres were similar for the three batches (Table 1), but the particle size distribution was skewed in favor of larger particles at the 20\% loading (Fig. 1a). This may reflect some aggregation of the odd shaped particles.

\section{Sudan III}

Sudan III loadings of $1 \%$ and $10 \%$ were studied with solvent evaporation under low vacuum conditions. At $1 \%$ loading, smooth microspheres devoid of surface pores were produced (Fig. 4a). These spheres released no drug within the one week test period. At the higher drug loading, some odd shaped particles were produced. Optical microscopy of the wet sample indicated that this was due to needle shaped crystals of Sudan III forming inside the polymer spheres, causing them to stretch along one or more axes. These leaf shaped particles are also evident from SEM (Fig. 4b). In addition, some 


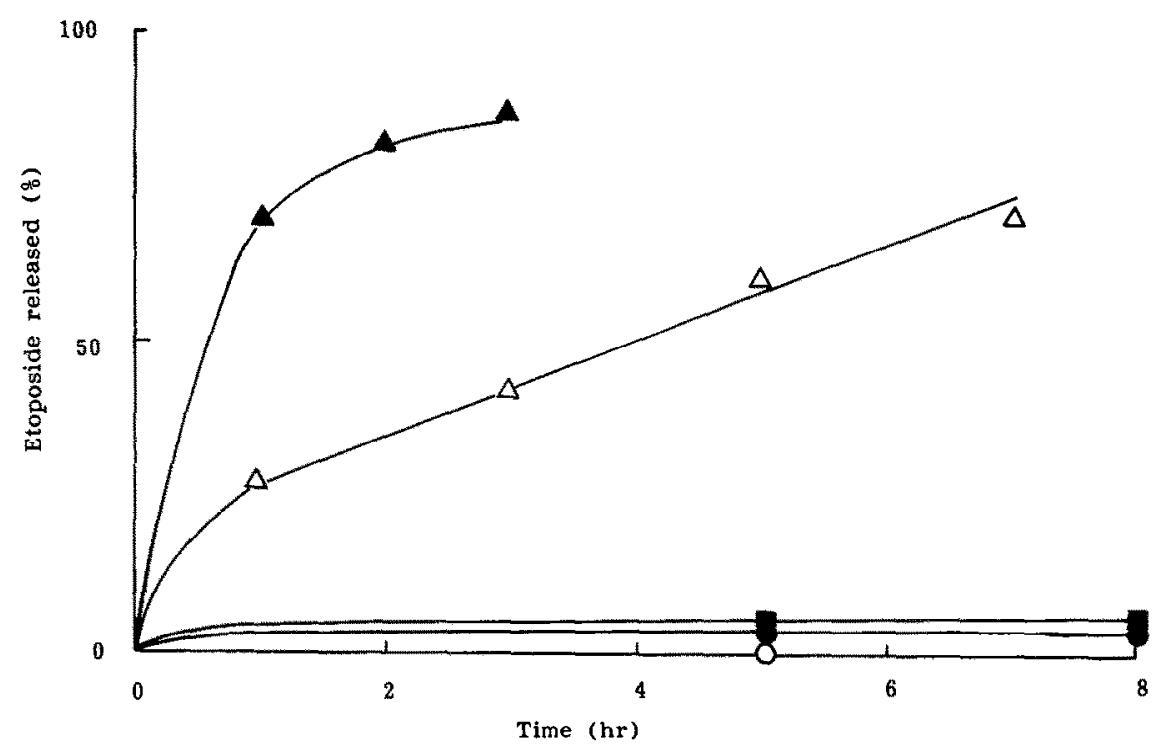

Fig. 2. Percentage release as a function of time for $P(L) L A$ microspheres containing etoposide. $(O) 1 \%$, low vac.; $(A) 10 \%$, atmospheric pressure; $(\mathbb{E}) 10 \%$, low vac.; $(\bullet) 10 \%$, high vac.; $(\Delta) 20 \%$, low vac.
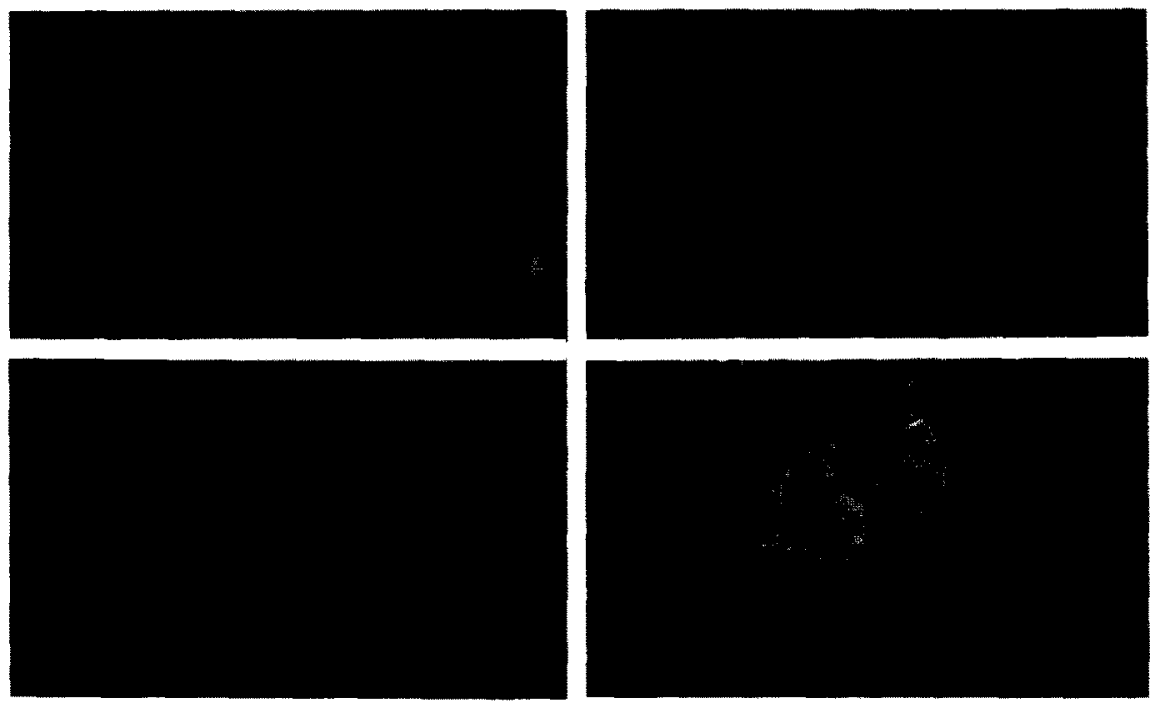

Fig. 3. Scanning electron micrographs of $P(L) L A$ microspheres containing etoposide. (a): $1 \%$, low vacuum; (b): 10\%, low vacuum; (c): $10 \%$, atmospheric pressure, and (d): $20 \%$, low vacuum.

of the spheres had rough surfaces and a small proportion of distorted microspheres was formed. In contrast to the case of etoposide, the presence of odd shaped microspheres did not result in any observable release of Sudan III within the test period. For both loadings of Su- dan III, assay of the $44-88 \mu \mathrm{m}$ particle size fraction indicated about the same degree of drug incorporation as a percentage of the theoretical value (Table 1), and no big difference was observed in the average particle size (Fig. 1b).

From these results it seems that drug loading 

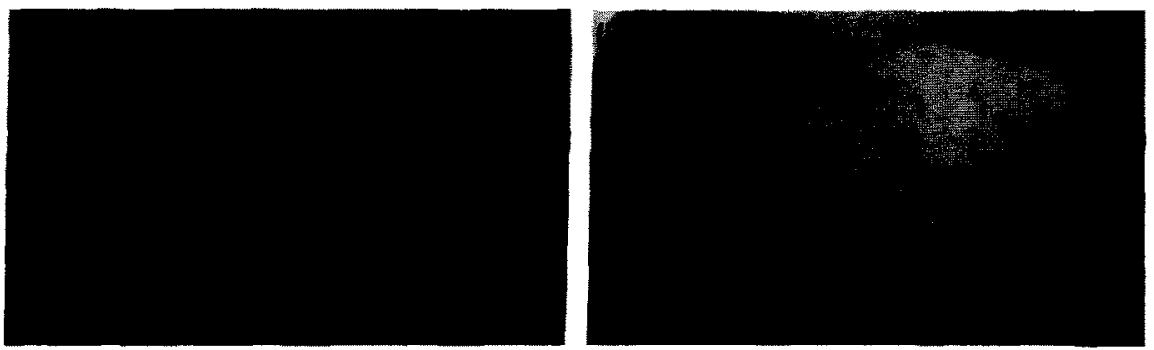

Fig. 4. Scanning electron micrographs of $P(L) L A$ microspheres containing Sudan III. (a): $1 \%$, low vacuum, and (b): 10, low vacuum.

is an important determinant of the morphology of the particles formed. For the relatively polar etoposide, the change in morphology was associated with a change in the release rate of the drug. For the highly lipophilic Sudan III, the change in morphology did not result in any release. The contrast between the results for the lipophilic and the polar compound suggests that drug lipophilicity is also an important factor in determining release from $\mathbf{P}(\mathrm{L}) \mathrm{LA}$ microspheres.

\section{Drug lipophilicity}

\section{At $1 \%$ loading}

In both cases, for etoposide and Sudan III batches with solvent evaporation under low vacuum conditions using $1 \%$ gelatin as the emulsifying agent, very smooth surfaced and consistently spherical particles were produced, and batches were free of distorted microspheres (Figs. $3 a$ and $4 a$ ). In neither case was any drug released during the test period (Fig. 2).

\section{At $10 \%$ loading}

Yields and particle size distributions were similar for batches containing etoposide or $\mathrm{Su}$ dan III at $10 \%$ loading (Table 1 and Fig. 1). Examination by SEM (Figs. $3 \mathrm{~b}$ and $4 \mathrm{~b}$ ) indicated that, for both drugs, the sample contained some rough surfaced particles and some distorted particles. Whereas the majority of etoposide particles were spherical, many of the $\mathrm{Su}$ dan III particles were ellipsoid or leaf shaped.
There was a small amount of drug released in the case of etoposide (Fig. 2) but no release of Sudan III was observed.

At low drug loading, smooth microspheres were formed irrespective of the drug lipophilicity, and no release was observed, for the batches with solvent evaporation under low vacuum conditions. At higher loading, the greater percentage of distorted particles produced may be due to drug precipitation during evaporation of the solvent. In the case of etoposide, higher amounts of distorted particles were associated with greater drug release, but this was not so for Sudan III. The drug lipophilicity thus appears to be of great importance in affecting the release rate when the drug loading is high.

\section{Solvent removal rate}

\section{Sudan III, 1\%}

In this region of drug lipophilicity and loading, smooth microspheres were produced under the usual solvent removal conditions when PVA was used as the emulsifying agent. Removing the solvent under a higher vacuum or at atmospheric pressure resulted in a similar yield, assay and particle size distribution. Changing the solvent removal rate had no effect on the particle shape; smooth surfaced, spherical particles were produced in each case. None of these batches released any Sudan III within the one week test period. In the case of the gelatin emulsion, however, the particle shape and the porosity were 
affected by the solvent evaporation rate (Table 1).

\section{Etoposide 10\%}

At this loading of etoposide, microspheres were smooth surfaced and well formed for the most part, with occasional surface roughness and some distorted particles under low vacuum conditions. When a high vacuum was used to remove the methylene chloride, the yield, particle size distribution, porosity and surface smoothness were almost identical (Table 1 and Fig. 1). Changing the solvent removal rate had no effect on the release pattern of etoposide; a small amount of etoposide was released almost immediately, followed by very slow release. Less than $10 \%$ was released within $8 \mathrm{~h}$. When the solvent was evaporated under atmospheric pressure conditions, the percentage of rough distorted microspheres with relatively large porosity was markedly increased (Fig. $3 \mathrm{c}$ ). As with the $20 \%$ etoposide loading, the high percentage of distorted particles corresponding to a fast release pattern, in this case $86 \%$ in $3 \mathrm{~h}$ (Fig. 2).

The results of these two series of experiments suggest that, in the case of the PVA emulsion, the solvent evaporation rate has little effect on morphology. By contrast, when gelatin is used as the emulsifying agent, the percentage of distorted microspheres is highly sensitive to the solvent evaporation rate.

\section{CONCLUSIONS}

These experiments show that drug lipophilicity, drug loading and solvent removal rate may all play an important role in determining the morphology and release rate from poly (L) lactic acid microspheres. Under conditions where some distorted microspheres are formed, the morphology is very sensitive to drug loading and solvent evaporation rate, while the release rate depends on the drug lipophilicity as well as the other two factors.

\section{REFERENCES}

1 T. Sato, M. Kanke, H.G. Schroeder and P.P. DeLuca, Porous biodegradable microspheres for controlled drug delivery. I. Assessment of processing conditions and solvent removal techniques, Pharm. Res., 5 (1988) 21-30.

2 R. Bodmeier and J.W. McGinity, The preparation and evaluation of drug-containing poly (D,L-lactide) microspheres formed by the solvent evaporation method, Pharm. Res., 4 (1987) 465-471.

3 M. Cavalier, J.P. Benoit and C. Thies, The formation and characterization of hydrocortisone-loaded poly $((+)$-lactide) microspheres, J. Pharm. Pharmacol., 38 (1986) 249-253.

4 A. Schindler, Y.M. Hibionada and C.G. Pitt, Aliphatic polyesters. III. Molecular weight and molecular weight distribution in alcohol-initiated polymerization of $\epsilon$ caprolactone, J. Polym. Sci., Polym. Chem. Ed., 20 (1982) 319-326.

5 Y. Aso, Y. Hayashi, S. Yoshioka, Y. Takeda, Y. Kita, Y. Nishimura and Y. Arita, Epimerization and hydrolysis of etoposide analogues in aqueous solution, Chem. Pharm. Bull., 37 (1989) 422-424. 